\title{
Estandarización del WISC-III en Chile: Descripción del Test, Estructura Factorial y Consistencia Interna de las Escalas
}

\section{Standarization of WISC-III in Chile: Test Description, Factorial Structure, and Internal Consistency of the Scales}

\author{
Valeria Ramírez y Ricardo Rosas \\ Pontificia Universidad Católica de Chile
}

\begin{abstract}
Se presentan los resultados iniciales del trabajo de adaptación y estandarización del WISC-III a la población chilena en base a la muestra de estandarización de 1924 niños obtenidos por medio de un muestreo aleatorio estratificado por regiones. Se presentan las principales diferencias con el WISC-R, la estructura factorial y consistencia interna de las escalas del WISC-III. Los resultados del estudio muestran una alta coincidencia con los de la prueba original norteamericana, obteniéndose cuatro factores de inteligencia y consistencias internas adecuadas a este tipo de pruebas. Sin embargo, las subpruebas incluidas en los factores difieren levemente de los resultados de la estandarización nortemericana.
\end{abstract}

Palabras Clave: WISC-III, estandarización de instrumentos.

\begin{abstract}
We present the results of the chilean adaptation and standarization of WISC-III. The sample for the study consisted in 1924 children obtained from a ramdomized stratified sampling considering the regions of Chile. Results show a great coincidence with those obtained in the original north-american sample: we obtained the same four intelligence factors and high internal consistencies. Nevertheless, the subscales included in the factors differ slightly from the north-american results.
\end{abstract}

Keywords: WISC-III, test estandarization.

Acerca de la Necesidad de Contar con una
Escala de Inteligencia Actualizada

En 1949 David Wechsler construyó la primera "Escala de Inteligencia de Wechsler para Niños”(WISC). Esta escala fue estandarizada en Chile en 1962 por Campazzo. En 1974, Wechsler revisó y actualizó la escala, la que fue conocida con el nombre de WISCR. Tuvo como propósito evaluar la inteligencia del niño y predecir su logro escolar (Kaufman, 1975). La revisión conservó lo máximo posible de la escala original, modificando o eliminando ítemes ambiguos, obsoletos, inadecuados o poco discriminativos. En-

Valeria Ramírez, Escuela de Psicología, Pontificia Universidad Católica de Chile.

Ricardo Rosas, Escuela de Psicología, Pontificia Universidad Católica de Chile.

La correspondencia relativa a este artículo debe ser dirigida a Valeria Ramírez al e-mail: dramirec@puc.cl

Este proyecto fue financiado por Fondecyt $N^{\circ} 1020951$

"Estandarización de la tercera versión del test de inteligencia de Wechsler (WISC-III) para la población infantojuvenil de Chile".

Agradecemos la valiosa colaboración de María de los Angeles Herane, asistente de investigación, y a todos los colegios, niños y evaluadores participantes del estudio. tre 1975 y 1980 la escala WISC-R fue adaptada y estandarizada para el Área Metropolitana de Santiago de Chile por tres grupos de investigación (Adriasola et al., 1976; Cañas et al., 1978; Calderón, Castillo, Mandujano, Pérez \& Purcell, 1980). En 1991 David Wechsler y colaboradores realizaron una segunda revisión y actualización de la Escala. La prueba fue actualizada y estandarizada para Estados Unidos para niños de 6 a 16 años 11 meses, lográndose la Escala WISC-III. Si bien esta escala mantiene las características fundamentales del WISC-R, proporciona datos normativos actuales y materiales, contenidos y procedimientos de administración actualizados (Wechsler, 1991/1997). El WISC-R es la prueba de inteligencia más ampliamente utilizada actualmente en Chile. Un sondeo realizado en 1996 en 21 colegios del Área Metropolitana de Santiago (Ramírez, Cumsille \& Simonetti, 1996) reveló que el $62 \%$ de ellos utiliza el WISC-R. Sin embargo, los avances tecnológicos, el auge de las comunicaciones, el desarrollo de los medios de comunicación de masas, las modificaciones curriculares del sistema educacional y el mayor acceso a la educación en la actualidad, han dejado obsoleto el WISC-R, puesto que los avances señalados han permitido que los niños tengan en la actualidad una 
mayor visión y conocimiento del mundo. Por ello, las pruebas de inteligencia requieren de una urgente actualización en nuestro país. Los psicólogos chilenos desde hace tiempo vienen reclamando la necesidad de estandarizar para Chile la nueva escala, pues su experiencia les ha mostrado la obsolescencia del WISC-R. Es más, en general los niños muestran un cuociente intelectual (C. I.) mayor que el que denota su real capacidad intelectual. Al respecto, en Estados Unidos diversas investigaciones muestran que en los mismos individuos, los C.I. total, verbal y manual son aproximadamente 5 puntos más bajos cuando se evalúa con el WISC-III que cuando se hace con el WISC$\mathrm{R}$, tanto en niños con discapacidades de aprendizaje como con niños sin discapacidades (Horn \& Michele, 1999).

Así como el equipo de Wechsler vio la necesidad de actualizar el test, dados los avances tecnológicos y educativos y una estimulación cada vez más prematura de los niños, los profesionales que utilizan esta prueba en Chile también han percibido su obsolescencia y reclamado la necesidad de su actualización y nueva estandarización. Es necesario señalar que la prueba ya lleva diez años de actualizada para la población infantil de Estados Unidos, mientras en Chile se utiliza una versión que data de 27 años atrás.

En el Código Ético de la American Psychological Association (1992), se señala que los psicólogos que realizan investigación con tests deben utilizar procedimientos científicos y conocimientos profesionales actualizados para el diseño de los tests, estandarización y validación. Asimismo, se señala que los psicólogos no deben basar sus evaluaciones, decisiones sobre la intervención o recomendaciones, en datos o resultados de los tests que están desfasados en relación con los objetivos perseguidos en la actualidad, ni en medidas o tests obsoletos, inapropiados para los objetivos actuales. Sin embargo, en Chile aún se utiliza una versión que puede ya no ser válida, por lo que la adaptación y estandarización del WISC-III se convierte así en un aspecto ético de la práctica profesional del psicólogo que aún utiliza la versión antigua. Debe señalarse que, en base a los resultados arrojados por el WISC-R, se toman importantes decisiones en el ámbito escolar y judicial. Muchos peritajes judiciales utilizan esta prueba.

Cuando se percibe que el test que está a disposición ya no evalúa lo que se pretende, o lo evalúa mal, los psicólogos se ven tentados a utilizar un test actualizado, aun cuando no esté estandarizado para la población con la cual trabajan. Esto es lo que está sucediendo con el WISC-III ya que, aun cuando no está estandarizado ni hay normas para Chile, se ha comenzado a utilizar con las normas establecidas para Estados Unidos. A pesar que el WISC-III trata de neutralizar las diferencias culturales, en muchas circunstancias no lo logra (Cayssials, 1998). Lo anterior implica, entonces, un segundo dilema ético.

Lo señalado anteriormente justifica, entonces, realizar una adaptación y estandarización del WISCIII para Chile, con el fin de contar con un instrumento válido y confiable que permita el diagnóstico adecuado de los niños chilenos del nuevo milenio.

\section{Acerca del Modelo de Wechsler, las escalas del Test de WISC y su Estructura Factorial}

El modelo teórico del test de inteligencia de Wechsler es un modelo factorial aditivo, pues corresponde a un enfoque global de la inteligencia. Según Wechsler, la inteligencia es un constructo hipotético y se refiere a la capacidad agregada o global de la persona para actuar con un propósito, pensar racionalmente e interactuar en forma efectiva con su medio ambiente (Matarazzo, 1972).

Wechsler señala que su escala de inteligencia no abarca toda la inteligencia y que algunas pruebas apelan a fenómenos no intelectuales, tales como la planificación y la conciencia de los objetivos, el entusiasmo, la dependencia y la independencia en cierto campo, la impulsividad, la ansiedad y la persistencia (Wechsler, 1991/1997). En este sentido, la concepción de Wechsler está en consonancia con lo señalado por Goleman (1996) respecto de la inteligencia emocional, la que incluiría autodominio, celo, persistencia y capacidad para automotivarse, entre otros aspectos.

En consideración a que la capacidad intelectual es sólo un aspecto de la inteligencia, Wechsler exhorta a las personas que interpretan los resultados de un test de inteligencia a distinguir entre el C.I. que arroja el test, por un lado, y la inteligencia, por otro (Wechsler, 1979, citado en Wechsler, 1991/1997).

El WISC-III contiene varias subpruebas, cada una de las cuales evalúa un aspecto diferente de la capacidad intelectual; pero el conjunto de subpruebas no abarca el conjunto de las facetas posibles de la inteligencia. Por ello, las entrevistas a los niños y sus padres pueden profundizar en los factores significativos que influyen no sólo en lo intelectual sino también en la conducta del niño. Matarazzo (1972) ha señalado también a los psicólo- 
gos la necesidad de considerar la historia de vida del niño (i.e., sus antecedentes sociales, lingüísticos y culturales; y su historia clínica) para realizar una evaluación completa.

El WISC-III consta de 13 subpruebas (una más que el WISC-R), de las cuales 6 son verbales y 7 manuales. Las subpruebas verbales son: Información, Analogías, Aritmética, Vocabulario, Comprensión y Retención de Dígitos. Las manuales corresponden a: Completación de Figuras, Ordenamiento de Historias, Construcción con Cubos, Ensamblaje de Objetos, Claves, Laberintos y Búsqueda de Símbolos (esta última fue agregada en la tercera versión).

Según Spearman, las habilidades pueden ser expresadas como función de dos factores, uno general (g) común a todas las habilidades, y otro específico (s) para cualquiera habilidad particular y siempre distinto de los demás factores (Matarazzo, 1972).

En el WISC-III cada subprueba mide ciertas habilidades que son compartidas con otra u otras y ciertas capacidades que son específicas de aquella.

Las habilidades compartidas y específicas que mide cada subprueba son (Kaufman, 1979/1982):

Completación de figuras. Alerta visual, memoria visual a largo plazo, evaluación, comprensión verbal, cognición, evaluación, organización perceptual, capacidad espacial, percepción visual de estímulos significativos, distinguir los detalles esenciales de los no esenciales, procesamiento holístico, organización visual sin actividad motora indispensable.

Información. Amplitud de la información adquirida en el hogar y la escuela, comprensión verbal, memoria a largo plazo.

Claves. Capacidad para seguir instrucciones, velocidad y agudeza en tareas rutinarias, velocidad psicomotora, memoria visual a corto plazo, evaluación, funcionamiento cerebral integrado, coordinación visomotora, secuenciación, independencia de la distracción, capacidad de aprendizaje, percepción visual de estímulos abstractos, producción convergente, reproducción de modelos, habilidad de lápiz y papel.

Analogías. Razonamiento lógico abstracto, comprensión verbal, cognición, expresión verbal, conceptualización verbal, pensamiento abstracto, razonamiento verbal, capacidad para distinguir los detalles esenciales de los no esenciales, formación de conceptos verbales.

Ordenamiento de historias. Secuenciación temporal, concepto de tiempo, anticipación de consecuencias, comprensión verbal, evaluación, organi- zación perceptual, funcionamiento cerebral integrado, razonamiento verbal, sentido común, juicio social, organización visual sin actividad motora indispensable, producción convergente, capacidad de planificación.

Aritmética. Razonamiento numérico, resolver problemas aritméticos planteados en palabras (resueltos mentalmente) comprensión verbal, cognición, conocimiento adquirido, memoria, secuenciación, facilidad con los números, independencia de la distracción, alerta mental.

Construcción con cubos. Análisis del todo en sus partes componentes, formación de conceptos no verbales, visualización espacial, cognición, evaluación, organización perceptual, funcionamiento cerebral integrado, capacidad espacial, coordinación visomotora, síntesis, percepción visual de estímulos abstractos, reproducción de modelos.

Vocabulario. Desarrollo del lenguaje, conocimiento de palabras, comprensión verbal, cognición, conocimiento adquirido, expresión verbal, conceptualización verbal, memoria a largo plazo, acopio de información, pensamiento abstracto, capacidad de aprendizaje.

Ensamblaje de objetos. Capacidad para aprovechar la retroalimentación sensorial motora, anticipación de las relaciones entre las partes, flexibilidad para trabajar en dirección a una meta determinada, cognición, evaluación, organización perceptual, capacidad espacial, coordinación visomotora, percepción visual de estímulos significativos, síntesis, procesamiento holístico.

Comprensión. Información práctica, evaluación y uso de experiencias previas, comprensión verbal, expresión verbal, conceptualización verbal, razonamiento verbal, sentido común, juicio social.

Búsqueda de símbolos. Discriminación visual de estímulos abstractos, velocidad y precisión, atención y concentración, memoria a corto plazo, flexibilidad cognitiva (Cayssials, 1998).

Retención de dígitos. Memoria auditiva a corto plazo, secuenciación, independencia de la distracción, facilidad con los números, alerta mental.

Laberintos. Seguir un patrón visual y previsión, cognición, organización perceptual, funcionamiento cerebral integrado, capacidad espacial, coordinación visomotora, razonamiento no verbal, capacidad de planificación, habilidad de lápiz y papel.

Las subpruebas de Retención de Dígitos, Laberintos y Búsqueda de Símbolos se consideran complementarias y opcionales, y no se utilizan para el cálculo del C.I. Sin embargo, se recomienda su uso 
para lograr un estudio más completo de la capacidad del niño. La subprueba Retención de Dígitos puede reemplazar a una subprueba verbal y Laberintos, a una manual, si alguna de ellas resulta invalidada o no puede ser administrada. Búsqueda de Símbolos sólo puede reemplazar a Claves (Wechsler, 1991/ 1997). Wechsler ha declarado que prefiere aplicar Laberintos en vez de Claves a los niños menores de 8 años, debido a su mayor confiabilidad en esas edades (Kaufman, 1979/1982) y por cuanto es la mejor medida de la habilidad de organización perceptual de niños de 6 y 7 años; sin embargo, Laberintos tiene muy baja confiabilidad en las edades 8 a 16 . No obstante, las investigaciones realizadas muestran la importancia de la prueba Claves en niños con daño neurológico o trastornos graves de aprendizaje, por lo que se recomienda aplicar esta subprueba cuando se sospeche de la existencia de dichos daños o trastornos, incluso a los niños de 6 y 7 años (Kaufman, 1979/1982).

El instrumento arroja tres puntajes de C.I.: uno Total, uno de la Escala Verbal y uno de la Escala Manual.

$\mathrm{Al}$ actualizar la prueba, el equipo de Wechsler trató de mantener una ligazón histórica con el WISC$\mathrm{R}$, conservando lo más posible del test anterior. Sin embargo, hubo modificaciones de varios ítemes y reemplazo de varios otros. También se actualizaron los materiales de los tests y los procedimientos. Las correlaciones entre ambas versiones han oscilado en Estados Unidos entre 0.81 para la Escala Manual y 0.90 para la Escala Verbal. A su vez, las correlaciones entre las subpruebas oscilaron entre 0.42 (en Ordenamiento de Historias) y 0.80 (en Información) (Wechsler, 1991/1997).

En la muestra de estandarización de Estados Unidos (2.200 estudiantes de 6 a 16 años 11 meses), la confiabilidad del WISC-III fue evaluada por medio de una bipartición de cada subprueba (excepto Claves y Búsqueda de Símbolos), para la muestra total y para cada rango de edad, calculando una correlación entre ambas partes y utilizando la corrección de Spearman-Brown. En la muestra total se obtuvo un coeficiente de confiabilidad de 0.96 en la Escala Total, 0.95 en la Escala Verbal y 0.91 en la Escala Manual. Los coeficientes promedio para las pruebas de la Escala Verbal en cada rango de edad oscilaron entre 0.92 y 0.96 y los de las pruebas de la Escala Manual, entre 0.89 y 0.94. En las subpruebas, solamente se obtuvieron 13 coeficientes bajo 0.70 , de un total de 121. Estos se dieron para algunas edades en Ensamblaje de Objetos y Laberintos Para las subpruebas de Claves y Búsqueda de Símbolos la confiabilidad se evaluó por medio de la estabilidad de las mismas, empleando el sistema de testretest, calculando una correlación lineal de Pearson entre los puntajes de ambos momentos: estos fueron 0.79 y 0.76 , respectivamente. Debe señalarse que en las Escalas Verbal y Manual los coeficientes de confiabilidad aumentaron levemente en el WISC-III con relación al WISC-R.

En cuanto a la validez de constructo, las correlaciones de las subpruebas de la Escala Verbal con esta última oscilaron entre 0.53 (Retención de dígitos) y 0.71 (Analogías); las de la Escala Manual, entre 0.27 (Claves) y 0.63 (Ensamblaje de objetos).

En el WISC-R se habían identificado tres factores que subyacen al test: Comprensión Verbal, Organización Perceptual y Ausencia de Distractibilidad. En la estandarización realizada en Chile también se encontraron los mismos factores, explicando el 84.8, 9.7 y $5.5 \%$ de la varianza común total, respectivamente. El factor Ausencia de Distractibilidad fue denominado así porque, según Kaufman (1975, citado en Wechsler, 1994/1997), las subpruebas que mayor peso tienen en él (Claves, Aritmética y Retención de Dígitos) suponen concentración y atención. Sin embargo, hasta ahora la interpretación de este factor es objeto de debate, por cuanto otros han señalado que son tareas que requieren capacidad de establecer secuencias (Bannatyne, 1974, citado en Wechsler, 1991/1997), o que son las más susceptibles a la ansiedad (Lutey, 1977, citado en Wechsler, 1991/1997), o que las subpruebas requieren capacidad cuantitativa o numérica (Osborne \& Lindsey, 1967, citado en Wechsler, 1991/1997) o que requieren procesos de ejecución (Wielkiewicz, 1990, citado en Wechsler, 1991/1997).

En la muestra estadounidense de estandarización del WISC-III se encontraron cuatro factores. Las subpruebas de Información, Analogías, Vocabulario y Comprensión componen el Factor Comprensión Verbal; las de Completación de Figuras, Ordenamiento de Historias, Construcción con Cubos y Ensamblaje de Objetos, componen el Factor Organización Perceptual; Aritmética y Retención de Dígitos, el Factor Ausencia de Distractibilidad; y Claves y Búsqueda de Símbolos, el cuarto Factor, Velocidad de Procesamiento. Los dos primeros tienen la misma composición que en el WISC-R, explicando aproximadamente el $45 \%$ de la varianza; el tercero, mostró una composición algo diferente (previamente estaba compuesto por Aritmética, Claves y Retención de dígitos) pero, por continuidad histórica, sigue 
denominándoselo Ausencia de Distractibilidad. El cuarto factor, que fue denominado Velocidad de Procesamiento, resultó por la inclusión de la subprueba Búsqueda de Símbolos, la que se aglomeró esta vez con Claves (Wechsler, 1991/1997).

Respecto de la validez de criterio del WISC-III, estudios realizados en Estados Unidos han demostrado una gran validez concurrente, dadas las altas correlaciones que ha mostrado con otras escalas con las que se esperaba que se correlacionara. Es el caso del WAIS-R ( $r=0.86$ en la Escala Total; 0.90 en la Escala Verbal; 0.80 en la Escala Manual); del WPPSI$\mathrm{R}(r=0.85$ en la Escala Total; 0.85 en la Escala Verbal; 0.73 en la Escala Manual); del Otis-Lennon School Ability Test $(r=0.73)$; y del Differential Ability Scales (DAS) de Elliot $(r=0.92$ entre la Escala Total del WISC-III y el subtest de Habilidad Conceptual General del DAS; 0.87 entre la Escala Verbal del WISCIII y el puntaje de Habilidad Verbal del DAS; 0.82 entre la Escala Manual del WISC-III y Habilidad Espacial del DAS y 0.78 con la Habilidad de Razonamiento No Verbal del DAS) (Wechsler, 1991/1997). Estas pruebas no se encuentran en Chile.

Además de su utilización en niños "normales", el WISC-III ha sido puesto a prueba en grupos clínicos y muestras de niños excepcionales. En un programa de Estados Unidos para niños dotados, los niños de la muestra mostraron puntajes altos, dentro del rango estipulado para niños dotados. Por su parte, niños con retardo mental han mostrado puntajes que caen en la categoría de retardo moderado del WISC-III. Otros estudios han mostrado evidencia de la validez del WISC-III para distinguir grupos de niños con discapacidades de aprendizaje e, incluso, con daños neuropsicológicos (Wechsler, 1991/1997). En Argentina, Cohen (1999) reporta una diferencia en la capacidad cognitiva de niños que han sufrido maltrato (físico, psíquico o sexual) en San Miguel de Tucumán, en el sentido que el 60\% de estos niños mostró un cuociente intelectual inferior a la media.

\section{Objetivos}

El presente trabajo tiene los siguientes objetivos:

Mostrar las principales características del proceso de adaptación y estandarización chilena del WISC-III.

Mostrar las principales diferencias del WISC-III con la versión chilena del WISC-R.
Mostrar los principales resultados del análisis de los datos normativos respecto a la estructura factorial y consistencia interna de las escalas del nuevo test.

\section{Método}

\section{Muestra}

En una primera fase del estudio se aplicó la versión del test argentino a una muestra de 198 niños de de 6 a 16 años de colegios municipales, particulares subvencionados y particulares pagados de la Región Metropolitana. A partir de los resultados obtenidos, se evaluó la pertinencia de algunos ítemes, que resultaron problemáticos, con una muestra de 10 jueces. Los jueces fueron psicólogos expertos en psicometría, en tests de diagnóstico, en evaluación y medición o con gran experiencia en la administración e interpretación del WISCR. De este estudio se obtuvo una versión adaptada del WISCIII, que es la que finalmente se estandarizó.

El diseño muestral de la estandarización fue probabilístico. La muestra fue por conglomerados y estratificada (según los estratos señalados), considerando 6 etapas: regiones del país, departamentos provinciales de educación, comunas, establecimientos educacionales, cursos, niños. Las regiones seleccionadas para el estudio fuerton la Metropolitana, cuarta, quinta y novena. La muestra final fue de 1914 niños, divididos en 11 categorías de edad (ver Tabla 1).

\section{Instrumento}

El instrumento escogido para el proceso de adaptación y estandarización, fue la versión española del Test, estandarizada en Argentina, editada y distribuida por Paidós, a sugerencia de los encargados de la Psychological Corporation. Dicho test fue adaptado en una primera etapa a la población chilena, difiriendo por lo tanto en algunos aspectos menores de la versión disponible comercialmente por Paidós. Las principales modificaciones al test original son objeto de otra publicación.

\section{Resultados}

Principales diferencias del WISC-III con el WISC-R

En la Tabla 2 se presentan las principales diferencias de la versión chilena del WISC III con el test actualmente en uso en Chile (WISC-R). Se indican para cada escala, los ítemes que han sido modificados, los ítemes nuevos, los ítemes eliminados del WISC-R, el grado de dificultad comparado en las escalas de los dos test y la extensión y puntajes máximos de ambos test.

Como dato general, es destacable que el nuevo test consta de una subprueba adicional (búsqueda de símbolos), y que las restantes se mantienen estructuralmente idénticas, aunque con importantes variaciones en sus contenidos.

Respecto del grado de dificultad de los ítemes del WISC$\mathrm{R}$ que se mantuvieron en el WISC-III, es destacable que este disminuye como norma general. Esto es consistente con estudios internacionales que tienden a mostrar una consistente disminución de la dificultad de los ítemes de las pruebas de inteligencia con el paso del tiempo, fenómeno conocido como efecto Flynn, en honor a su descubridor, James Flynn 
Tabla 1

Distribución de la muestra, según edad, NSE y sexo. Total y regiones.

\begin{tabular}{|c|c|c|c|c|c|c|c|c|c|c|}
\hline \multirow{3}{*}{$\begin{array}{l}\text { TOTALY } \\
\text { REGIÓN }\end{array}$} & \multirow{3}{*}{ EDAD } & \multicolumn{6}{|c|}{ NSE } & \multirow{2}{*}{\multicolumn{3}{|c|}{ TOTAL }} \\
\hline & & \multicolumn{2}{|c|}{ Bajo } & \multicolumn{2}{|c|}{ Medio } & \multicolumn{2}{|c|}{ Alto } & & & \\
\hline & & $\mathrm{H}$ & M & $\mathrm{H}$ & M & $\mathrm{H}$ & M & $\mathrm{H}$ & M & Total \\
\hline \multicolumn{11}{|l|}{ TOTAL } \\
\hline & $6.0-6.6$ & 15 & 14 & 15 & 18 & 14 & 13 & 44 & 45 & 89 \\
\hline & $6.7-6.11$ & 14 & 15 & 15 & 12 & 15 & 16 & 44 & 43 & 87 \\
\hline & 7.0-7.6 & 15 & 11 & 15 & 15 & 12 & 18 & 42 & 44 & 86 \\
\hline & $7.7-7.11$ & 15 & 18 & 12 & 13 & 17 & 12 & 44 & 43 & 87 \\
\hline & 8 & 29 & 29 & 30 & 29 & 29 & 29 & 88 & 87 & 175 \\
\hline & 9 & 29 & 29 & 27 & 29 & 30 & 29 & 86 & 87 & 173 \\
\hline & 10 & 29 & 29 & 30 & 28 & 29 & 29 & 88 & 86 & 174 \\
\hline & 11 & 29 & 29 & 27 & 30 & 29 & 29 & 85 & 88 & 173 \\
\hline & 12 & 28 & 30 & 31 & 29 & 29 & 28 & 88 & 87 & 175 \\
\hline & 13 & 30 & 30 & 30 & 30 & 28 & 30 & 88 & 90 & 178 \\
\hline & 14 & 29 & 29 & 29 & 28 & 29 & 29 & 87 & 86 & 173 \\
\hline & 15 & 31 & 30 & 30 & 30 & 29 & 29 & 90 & 89 & 179 \\
\hline & 16 & 29 & 29 & 29 & 29 & 29 & 30 & 87 & 88 & 175 \\
\hline & Total & 322 & 322 & 320 & 320 & 319 & 321 & 961 & 963 & 1924 \\
\hline \multicolumn{11}{|c|}{ Región Metrop. } \\
\hline & $6.0-6.6$ & 6 & 6 & 5 & 7 & 9 & 7 & 20 & 20 & 40 \\
\hline & $6.7-6.11$ & 5 & 6 & 6 & 5 & 8 & 9 & 19 & 20 & 39 \\
\hline & $7.0-7.6$ & 6 & 5 & 6 & 7 & 5 & 6 & 17 & 18 & 35 \\
\hline & $7.7-7.11$ & 6 & 6 & 5 & 5 & 10 & 7 & 21 & 18 & 39 \\
\hline & 8 & 12 & 11 & 11 & 12 & 13 & 14 & 36 & 37 & 73 \\
\hline & 9 & 11 & 12 & 11 & 11 & 15 & 13 & 37 & 36 & 73 \\
\hline & 10 & 11 & 11 & 12 & 11 & 13 & 15 & 36 & 37 & 73 \\
\hline & 11 & 12 & 11 & 10 & 12 & 12 & 12 & 34 & 35 & 69 \\
\hline & 12 & 11 & 12 & 11 & 11 & 12 & 13 & 34 & 36 & 70 \\
\hline & 13 & 11 & 11 & 12 & 11 & 12 & 13 & 35 & 35 & 70 \\
\hline & 14 & 12 & 11 & 11 & 12 & 10 & 12 & 33 & 35 & 68 \\
\hline & 15 & 11 & 12 & 11 & 11 & 12 & 11 & 34 & 34 & 68 \\
\hline & 16 & 11 & 11 & 12 & 11 & 10 & 13 & 33 & 35 & 68 \\
\hline & Total & 125 & 125 & 123 & 126 & 141 & 145 & 389 & 396 & 785 \\
\hline \multicolumn{11}{|l|}{ IV Región } \\
\hline & $6.0-6.6$ & 2 & 3 & 3 & 4 & 2 & 2 & 7 & 9 & 16 \\
\hline & $6.7-6.11$ & 3 & 2 & 3 & 1 & 3 & 4 & 9 & 7 & 16 \\
\hline & $7.0-7.6$ & 2 & 1 & 1 & 1 & 3 & 5 & 6 & 7 & 13 \\
\hline & 7.7-7.11 & 3 & 5 & 2 & 3 & 3 & 0 & 8 & 8 & 16 \\
\hline & 8 & 5 & 5 & 6 & 5 & 6 & 6 & 17 & 16 & 33 \\
\hline & 9 & 5 & 5 & 5 & 5 & 5 & 5 & 15 & 15 & 30 \\
\hline & 10 & 6 & 6 & 5 & 5 & 5 & 5 & 16 & 16 & 32 \\
\hline & 11 & 5 & 5 & 6 & 5 & 6 & 6 & 17 & 16 & 33 \\
\hline & 12 & 5 & 5 & 7 & 7 & 5 & 5 & 17 & 17 & 34 \\
\hline & 13 & 6 & 7 & 5 & 5 & 5 & 5 & 16 & 17 & 33 \\
\hline & 14 & 5 & 5 & 5 & 4 & 6 & 6 & 16 & 15 & 31 \\
\hline & 15 & 5 & 4 & 7 & 7 & 5 & 5 & 17 & 16 & 33 \\
\hline & 16 & 6 & 6 & 5 & 5 & 5 & 5 & 16 & 16 & 32 \\
\hline & Total & 58 & 59 & 60 & 57 & 59 & 59 & 177 & 175 & 352 \\
\hline
\end{tabular}




\begin{tabular}{|c|c|c|c|c|c|c|c|c|c|c|}
\hline \multirow{3}{*}{$\begin{array}{l}\text { TOTALY } \\
\text { REGIÓN }\end{array}$} & \multirow{3}{*}{ EDAD } & \multicolumn{6}{|c|}{ NSE } & \multirow{2}{*}{\multicolumn{3}{|c|}{ TOTAL }} \\
\hline & & \multicolumn{2}{|c|}{ Bajo } & \multicolumn{2}{|c|}{ Medio } & \multicolumn{2}{|c|}{ Alto } & & & \\
\hline & & $\mathrm{H}$ & $\mathrm{M}$ & $\mathrm{H}$ & M & $\mathrm{H}$ & $\mathrm{M}$ & $\mathrm{H}$ & M & Total \\
\hline \multicolumn{11}{|l|}{$V$ Región } \\
\hline & $6.0-6.6$ & 5 & 4 & 5 & 5 & 2 & 2 & 12 & 11 & 23 \\
\hline & $6.7-6.11$ & 4 & 5 & 4 & 4 & 2 & 2 & 10 & 11 & 21 \\
\hline & 7.0-7.6 & 4 & 4 & 6 & 6 & 2 & 5 & 12 & 15 & 27 \\
\hline & $7.7-7.11$ & 5 & 5 & 3 & 3 & 3 & 2 & 11 & 10 & 21 \\
\hline & 8 & 9 & 9 & 9 & 9 & 7 & 6 & 25 & 24 & 49 \\
\hline & 9 & 9 & 9 & 9 & 10 & 6 & 7 & 24 & 26 & 50 \\
\hline & 10 & 9 & 9 & 9 & 8 & 7 & 6 & 25 & 23 & 48 \\
\hline & 11 & 9 & 9 & 7 & 10 & 8 & 8 & 24 & 27 & 51 \\
\hline & 12 & 9 & 9 & 10 & 8 & 9 & 7 & 28 & 24 & 52 \\
\hline & 13 & 9 & 9 & 10 & 9 & 8 & 8 & 27 & 26 & 53 \\
\hline & 14 & 9 & 9 & 9 & 9 & 10 & 8 & 28 & 26 & 54 \\
\hline & 15 & 9 & 9 & 9 & 9 & 9 & 9 & 27 & 27 & 54 \\
\hline & 16 & 9 & 9 & 9 & 9 & 10 & 8 & 28 & 26 & 54 \\
\hline & Total & 99 & 99 & 99 & 99 & 83 & 78 & 281 & 276 & 557 \\
\hline \multicolumn{11}{|l|}{ IX Región } \\
\hline & $6.0-6.6$ & 2 & 1 & 2 & 2 & 1 & 2 & 5 & 5 & 10 \\
\hline & $6.7-6.11$ & 2 & 2 & 2 & 2 & 2 & 1 & 6 & 5 & 11 \\
\hline & 7.0-7.6 & 3 & 1 & 2 & 1 & 2 & 2 & 7 & 4 & 11 \\
\hline & $7.7-7.11$ & 1 & 2 & 2 & 2 & 1 & 3 & 4 & 7 & 11 \\
\hline & 8 & 3 & 4 & 4 & 3 & 3 & 3 & 10 & 10 & 20 \\
\hline & 9 & 4 & 3 & 2 & 3 & 4 & 4 & 10 & 10 & 20 \\
\hline & 10 & 3 & 3 & 4 & 4 & 4 & 3 & 11 & 10 & 21 \\
\hline & 11 & 3 & 4 & 4 & 3 & 3 & 3 & 10 & 10 & 20 \\
\hline & 12 & 3 & 4 & 3 & 3 & 3 & 3 & 9 & 10 & 19 \\
\hline & 13 & 4 & 3 & 3 & 5 & 3 & 4 & 10 & 12 & 22 \\
\hline & 14 & 3 & 4 & 4 & 3 & 3 & 3 & 10 & 10 & 20 \\
\hline & 15 & 6 & 5 & 3 & 3 & 3 & 4 & 12 & 12 & 24 \\
\hline & 16 & 3 & 3 & 3 & 4 & 4 & 4 & 10 & 11 & 21 \\
\hline & Total & 40 & 39 & 38 & 38 & 36 & 39 & 114 & 116 & 230 \\
\hline
\end{tabular}

$\mathrm{H}:$ hombres

M: mujeres

(1994, 1999). En su prolijo estudio de los puntajes de C.I. para diferentes poblaciones en un lapso de 60 años, este autor descubrió que los puntajes verdaderos de C.I. se incrementan de una generación a la siguiente en todos los países para los cuales se cuenta con datos.

\section{Estructura Factorial del WISC III}

Para dar cuenta de la validez de constructo de la prueba WISC III en la población chilena, se llevó a cabo un análisis factorial confirmatorio. Se realizaron análisis para la muestra total, y en cuatro grupos etarios relevantes: 6-7, 8-10, 11-13 y 14-16 años. Se propuso un modelo teórico, el cual fue estimado y comparado con dos modelos anidados alternativos utilizando el programa LISREL-8.7.
En base a la teoría e investigación disponible, particularmente los análisis factoriales realizados en USA y Argentina (Wechsler, 1991/1997), indican que la solución de cuatro factores correlacionados sería la más adecuada para dar cuenta de la estructura del test. La única diferencia entre ambas soluciones, es la presencia del indicador Laberintos en el segundo factor de la muestra Argentina, indicador que no figura en la solución factorial estadounidense.

El modelo teórico a contrastar, esta compuesto por:

Factor I. ("Comprensión Verbal"). Abarca las subpruebas de Información, Analogías, Vocabulario y Comprensión.

Factor II. ("Organización perceptual"). Comprende las subpruebas Completación de figuras, Ordenamiento de Historias, Construcción con Cubos, Composición de Objetos (Ensamblaje) y Laberintos. 


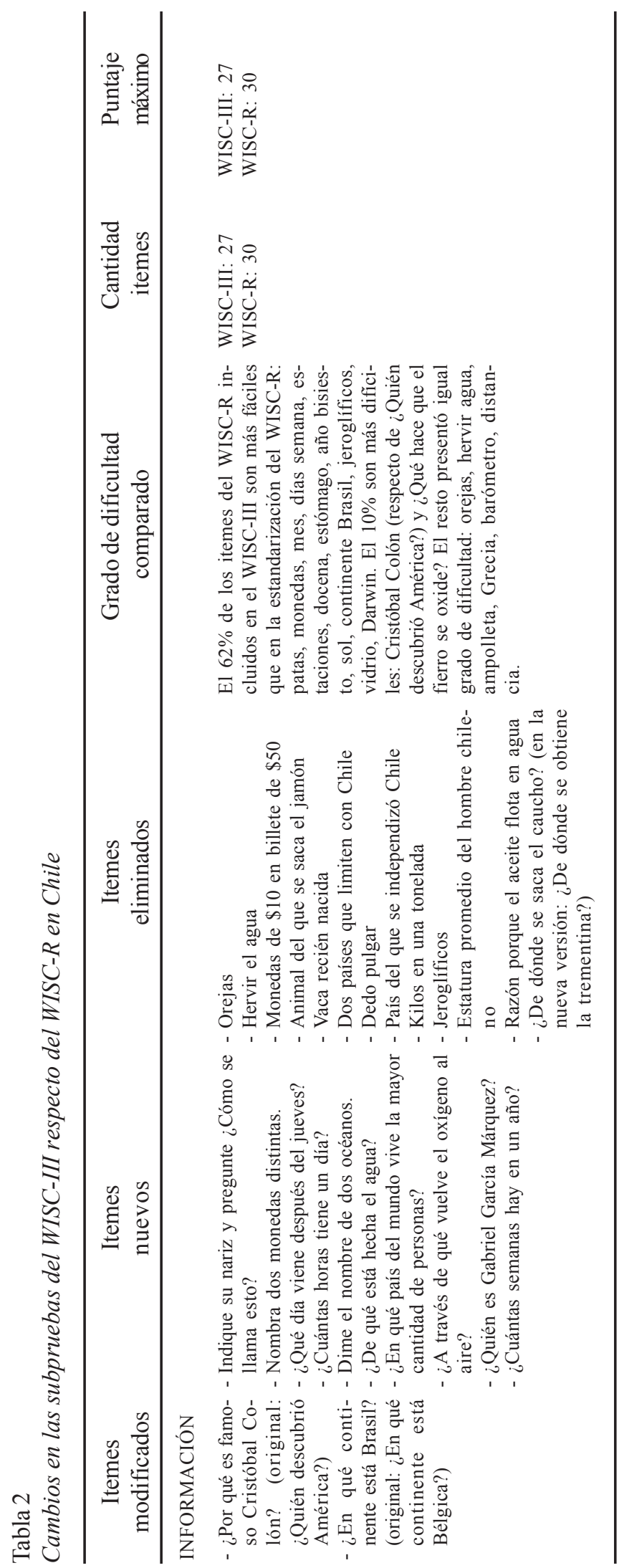

용

$\ddot{\exists} \ddot{\forall}$

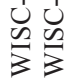

$\stackrel{\infty}{\rightarrow}=$

$\ddot{\exists} \ddot{\exists}$

$\stackrel{n}{\infty}$

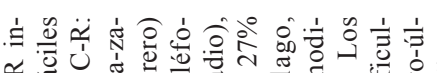

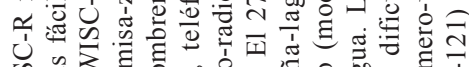

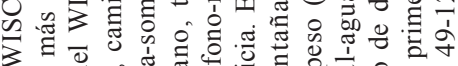

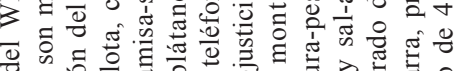

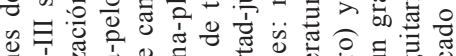

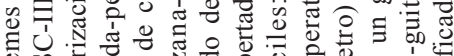

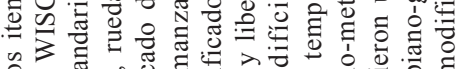

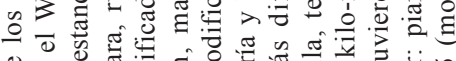
ช ฮ

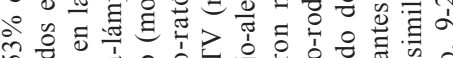

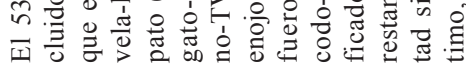
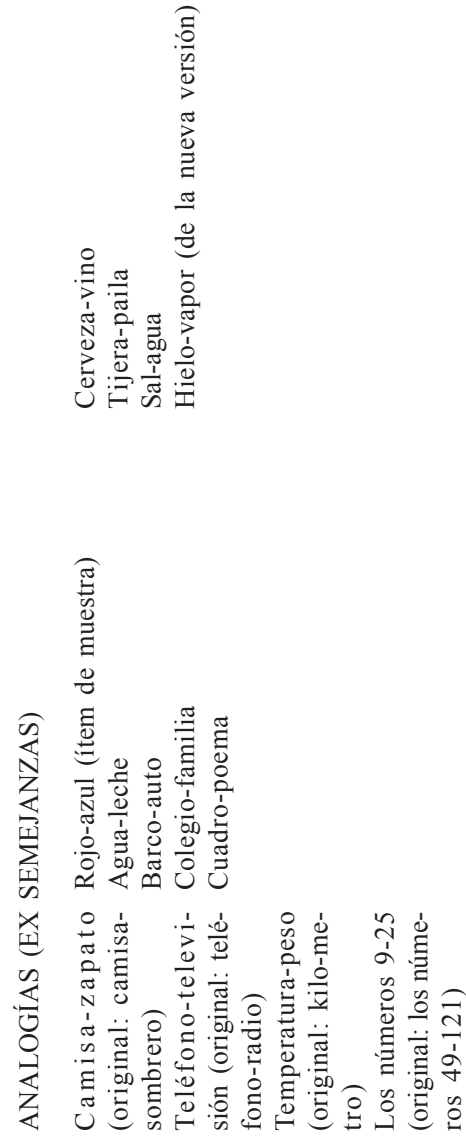


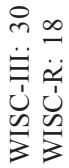

กิ

$\ddot{\exists}$

$\sum_{\substack{n \\ n}}^{n}$

$\approx \infty$

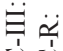

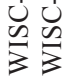

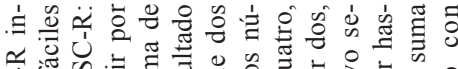

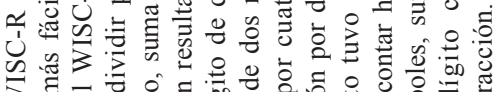

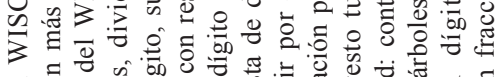

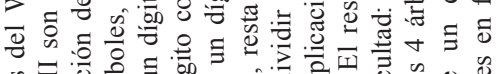

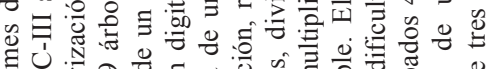

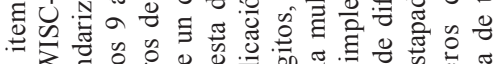

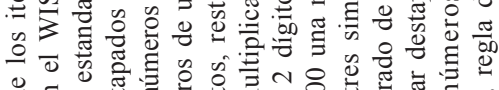

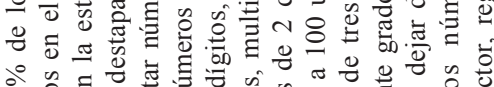

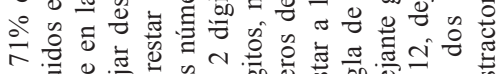

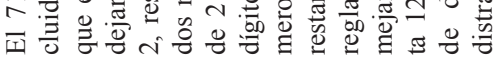

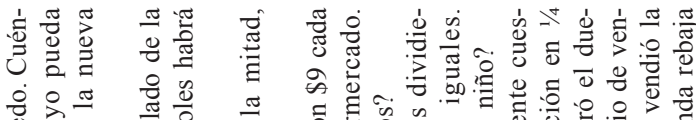

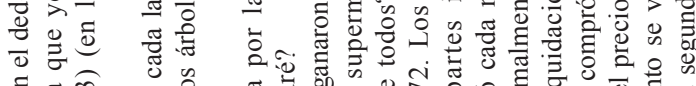

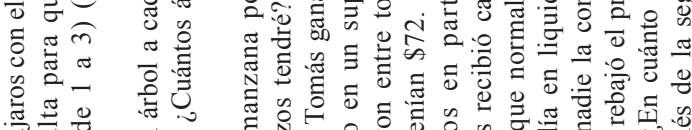

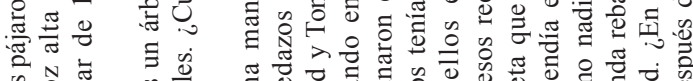

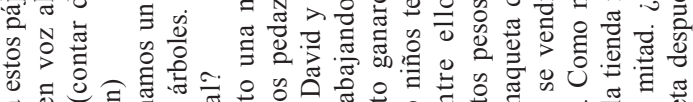

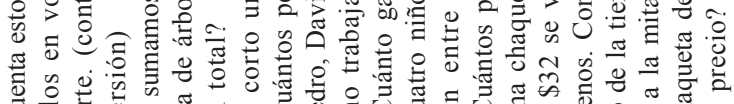

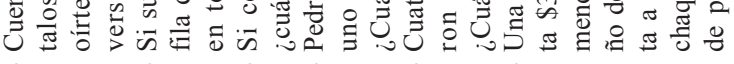

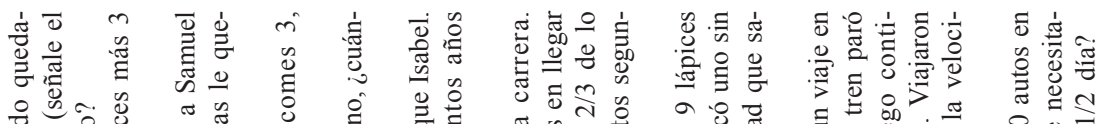

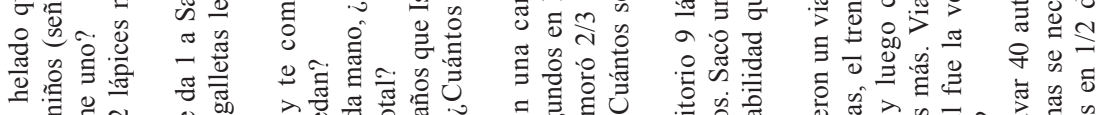

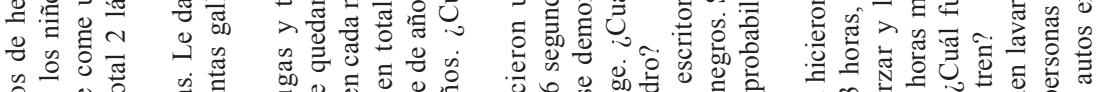

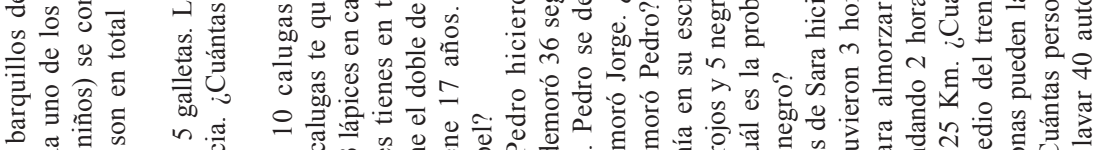

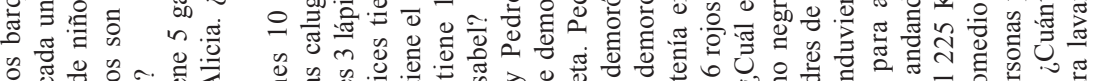

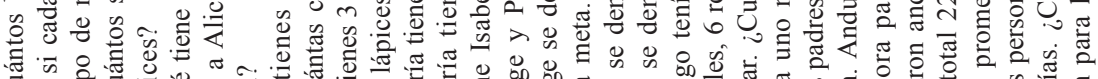

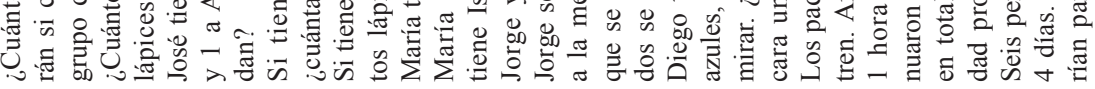

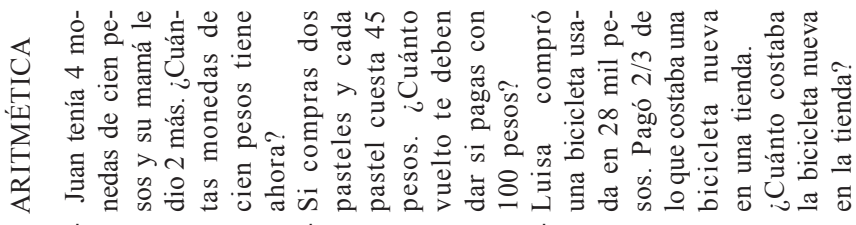

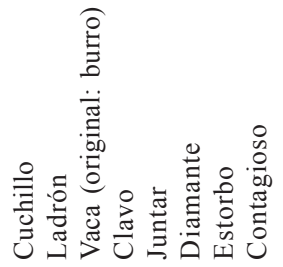

$\stackrel{\sim}{2}$

$\ddot{\exists}$

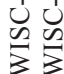

$\leqq \ddot{0}$

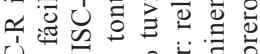

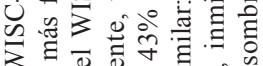

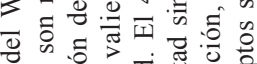

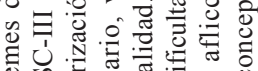

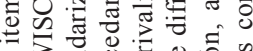

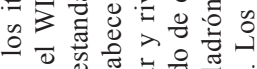

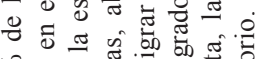

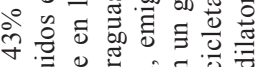

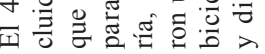

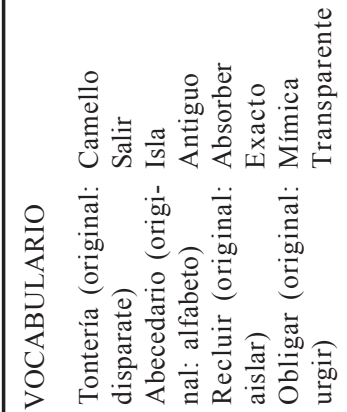




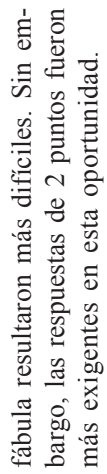

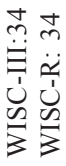

I

岁

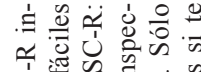

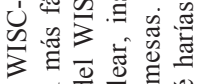

क्ष

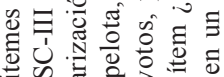

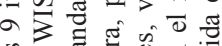

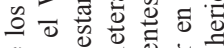

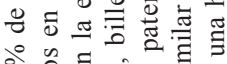

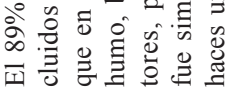

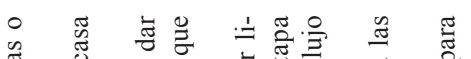

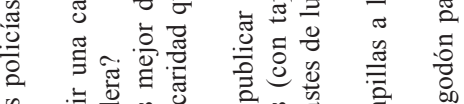

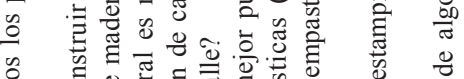

ठี ญ

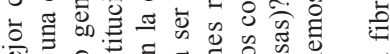

Ð

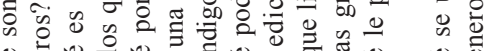

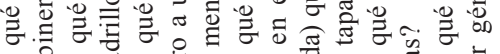

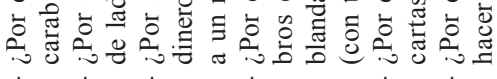

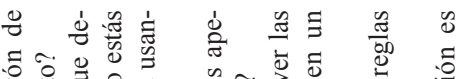

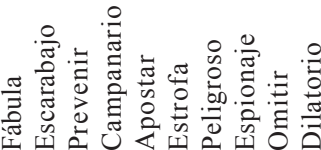

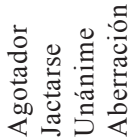

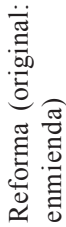

$\pm \pm \pm$

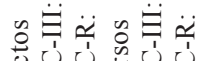

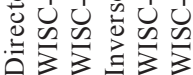

in

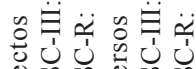

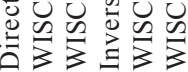

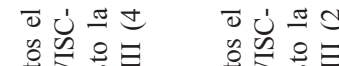

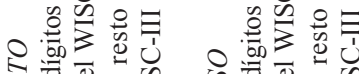

ठ

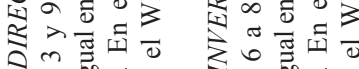

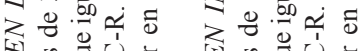

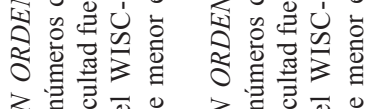

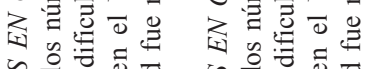

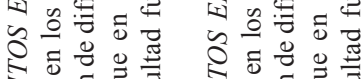

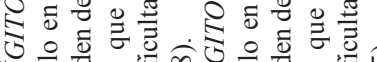

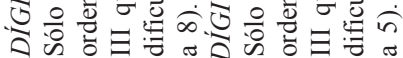


ลิ ธ

当

记

ํํำ

$\ddot{\exists}$

象

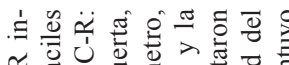

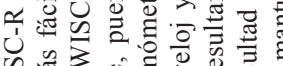

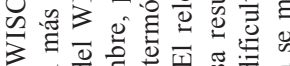

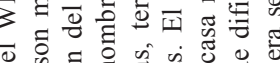

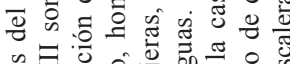

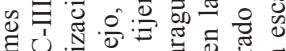
可

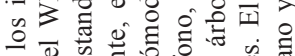

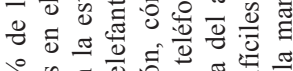

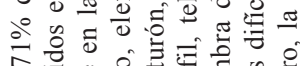

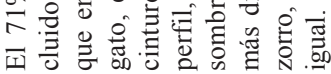

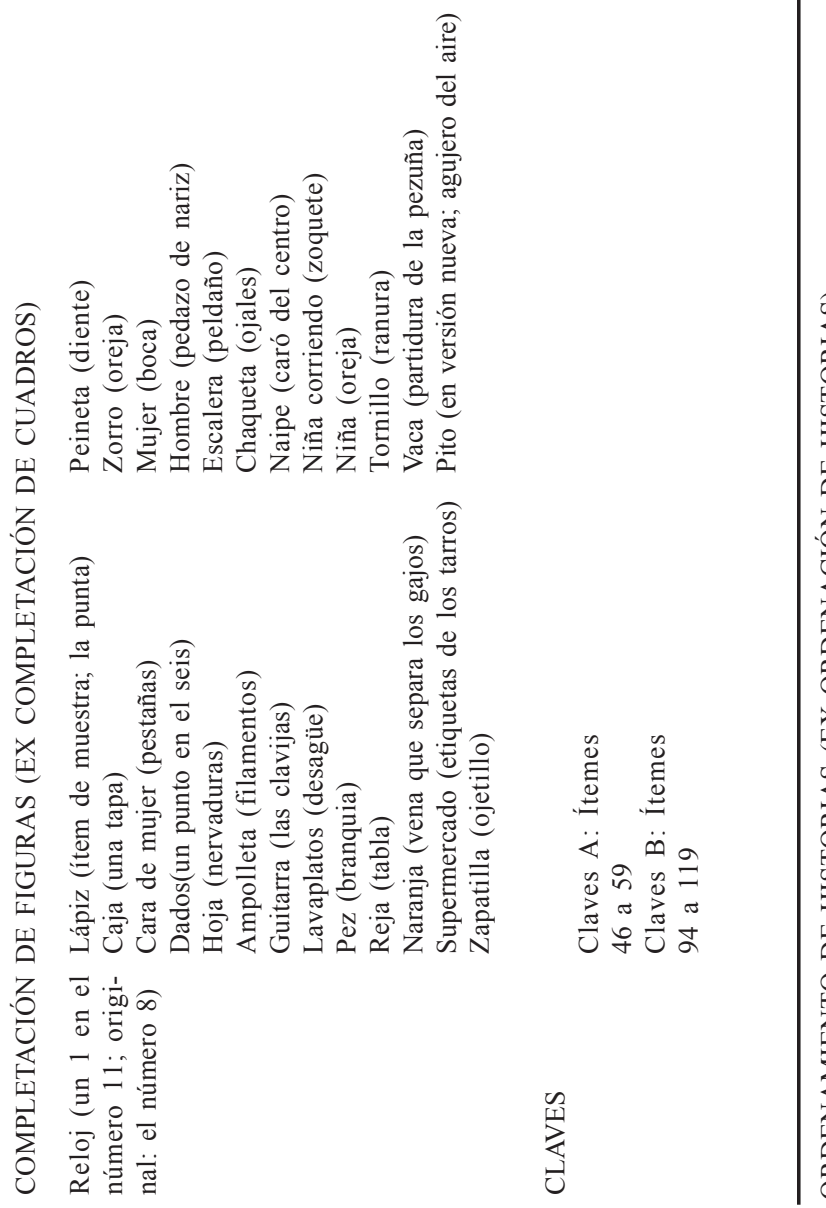

in

$\ddot{\exists}$

记

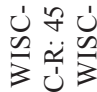

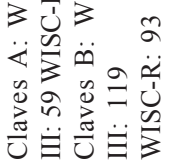

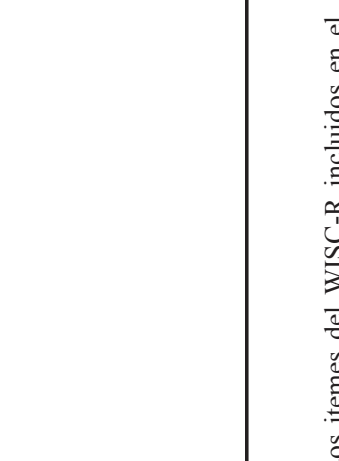

$\stackrel{m}{\longrightarrow}$

$\ddot{\ddot{\theta}} \ddot{\sim}$

淧
จิ

$\ddot{\exists}$

记

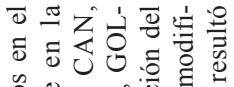

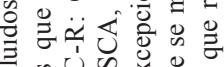

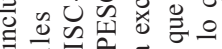

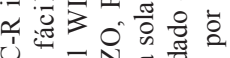

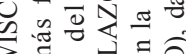

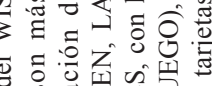

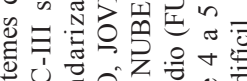

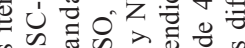

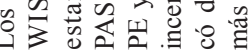

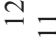

$\ddot{\exists}$

象

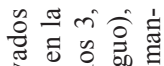

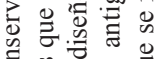

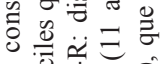

航

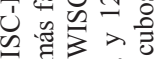

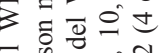

ฮ

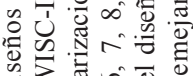

焉 60

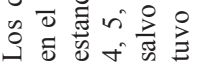

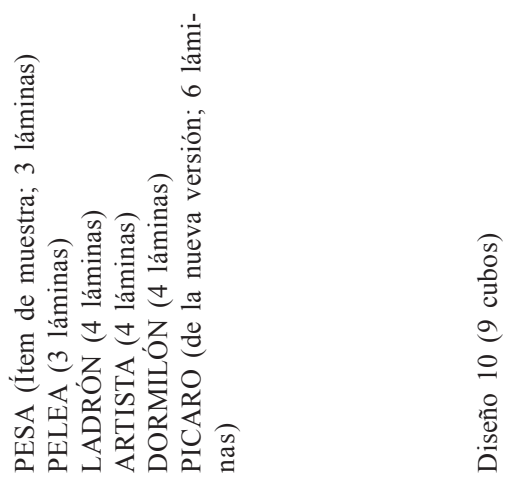

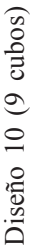

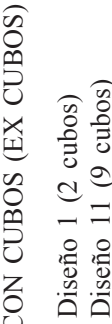

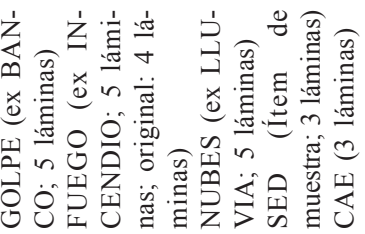

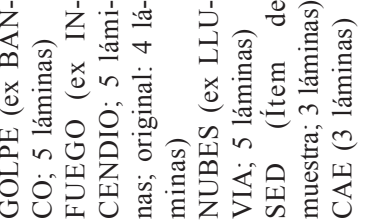




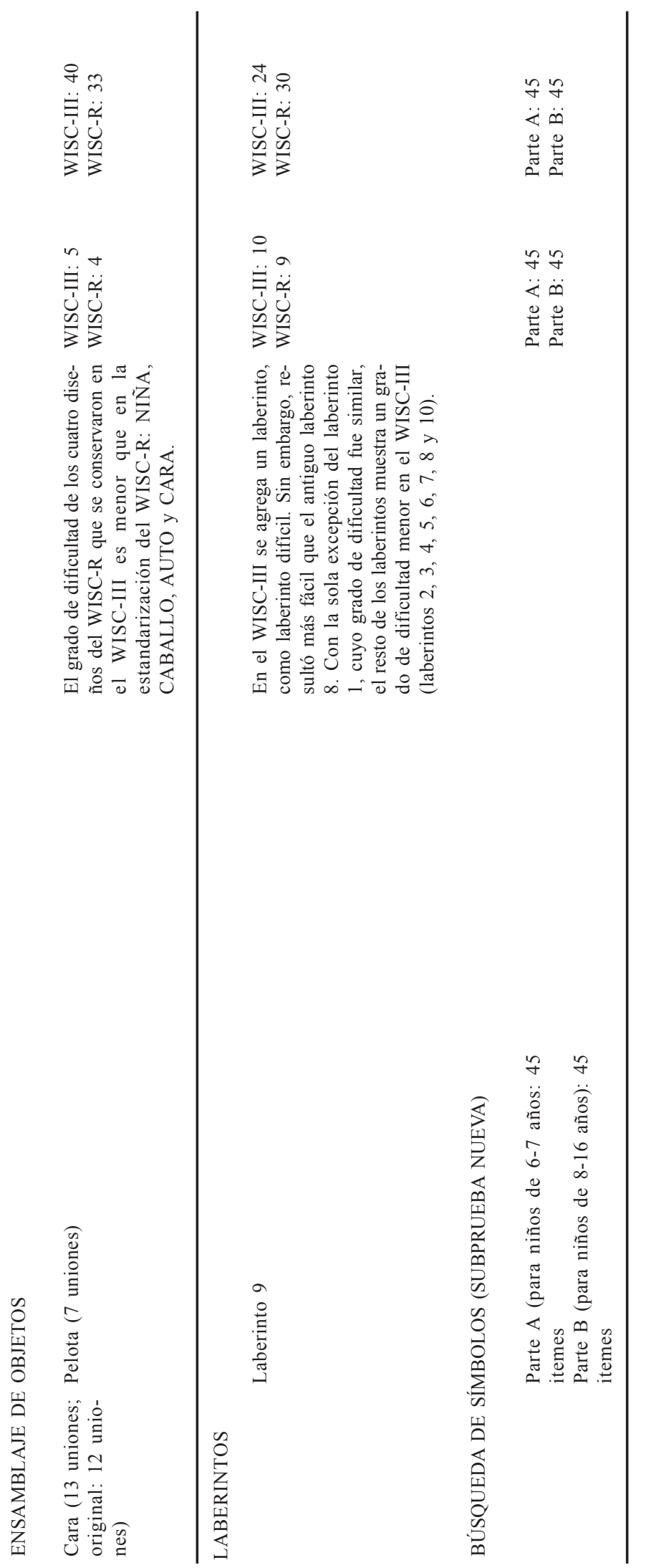


Factor III. ("Velocidad de Procesamiento"). Incluye las subpruebas de Claves y Símbolos.

Factor IV. ("Ausencia de Distractibilidad"). Incluye las subpruebas de Dígitos y Aritmética.

Los indicadores de ajuste (Tabla 3) muestran que el modelo propuesto posee un buen ajuste dado el tamaño muestral (modelo 4 factores $\chi 2(59)=392.91, p=0.000$, RMSEA $=$
0.054). Además, su índice de error de aproximación cuadrático medio (RMSEA) es el único que se encuentra en el rango aceptable, y el parámetro de no centralidad a escala (SNCF) es el más pequeño de los 4 modelos (Hair, Anderson, Tatham \& Black, 2004).

La comparación estadística de los modelos anidados se realizó comparando las diferencias entre los valores de $\chi 2$, considerando que estas diferencias se distribuyen como $\chi 2$

Tabla 3

Índices de ajuste y prueba de cambio en ajuste en modelos alternativos

\begin{tabular}{ccccccccc}
\hline Modelo & \multicolumn{4}{c}{ Índices de Ajuste } & \multicolumn{3}{c}{ Comparación $^{\mathrm{a}}$} \\
\hline & $\chi^{2}$ & $g l$ & $p$ & SNCP & RMSEA & $\Delta \chi^{2}$ & $g l$ & $p$ \\
1 factor & 1396.00 & 65 & 0.000 & 0.69 & 0.103 & - & & \\
2 factores & 761.47 & 64 & 0.000 & 0.36 & 0.075 & 643.53 & 1 & 0.000 \\
4 factores & 392.91 & 59 & 0.000 & 0.17 & 0.054 & 368.56 & 5 & 0.000 \\
\hline
\end{tabular}

a: Cada modelo se compara con el modelo de previo.

Tabla 4

Parámetros de la solución completamente estandarizada. Modelo factorial de la muestra total

\begin{tabular}{|c|c|c|c|c|c|}
\hline Factor & I & II & III & IV & \\
\hline Cargas Factoriales & & & & & $\mathrm{R}^{2}$ \\
\hline Información & $.78 * * *$ & & & & .61 \\
\hline Analogías & $.80 * * *$ & & & & .64 \\
\hline Vocabulario & $.80 * * *$ & & & & .64 \\
\hline Comprensión & $.64 * * *$ & & & & .41 \\
\hline Completación Figuras & & $.59 * * *$ & & & .35 \\
\hline Ordenamiento Historias & & $.63 * * *$ & & & .40 \\
\hline Construcción Cubos & & $.76 * * *$ & & & .57 \\
\hline Ensamblaje Objetos & & $.71 * * *$ & & & .50 \\
\hline Laberintos & & $.42 * * *$ & & & .17 \\
\hline Claves & & & $.61 * * *$ & & .38 \\
\hline Búsqueda Símbolos & & & $.78 * * *$ & & .61 \\
\hline Retención Dígitos & & & & $.55^{* * *}$ & .30 \\
\hline Aritmética & & & & $.75^{* * *}$ & .56 \\
\hline \multicolumn{6}{|c|}{ Correlaciones entre Factores } \\
\hline I & 1 & & & & \\
\hline II & $0.72 * * *$ & 1 & & & \\
\hline III & $0.51 * * *$ & $0.65^{* * *}$ & 1 & & \\
\hline IV & $0.81 * * *$ & $0.74 * * *$ & $.62 * * *$ & 1 & \\
\hline
\end{tabular}

$* * * p<.001$ 
Tabla 5

Índices de ajuste absoluto e incremental del modelo ${ }^{a}$, según edad

\begin{tabular}{ccccccccc}
\hline \multicolumn{9}{c}{ Absoluto } \\
\hline Edades & $\chi^{2}$ & $g l$ & $p$ & RMSEA & SNCF & NFI & TLI & Incremental \\
\hline $6-7$ & 132.41 & 59 & 0.000 & 0.060 & 0.21 & .92 & .94 & 349 \\
$8-10$ & 150.03 & 59 & 0.000 & 0.055 & 0.17 & .98 & .98 & 521 \\
$11-13$ & 139.12 & 59 & 0.000 & 0.051 & 0.15 & .94 & .95 & 527 \\
$14-16$ & 154.50 & 59 & 0.000 & 0.055 & 0.18 & .94 & .95 & 527 \\
\hline
\end{tabular}

a Modelo 4 factores.

Tabla 6

Parámetros de la solución completamente estandarizada. Modelo factorial de la muestra 6 a 7 años

\begin{tabular}{|c|c|c|c|c|c|}
\hline Factor & I & II & III & IV & $R^{2}$ \\
\hline Información & $.76 * * *$ & & & & .58 \\
\hline Analogías & $.78 * * *$ & & & & .61 \\
\hline Vocabulario & $.74 * * *$ & & & & .55 \\
\hline Comprensión & $.65 * * *$ & & & & .43 \\
\hline Completación 1 & & $.66^{* * *}$ & & & .44 \\
\hline Ordenamiento & & $.62 * * *$ & & & .38 \\
\hline Construcción & & $.71 * * *$ & & & .50 \\
\hline Ensamblaje $\mathrm{Ob}$ & & $.68 * * *$ & & & .46 \\
\hline Laberintos & & $.53 * * *$ & & & .29 \\
\hline Claves & & $.49 * * *$ & & .24 & \\
\hline Búsqueda Sím & & & $.82 * * *$ & & .67 \\
\hline Retención Dígi & & & & $.65^{* * *}$ & .43 \\
\hline Aritmética & & & & $.73 * * *$ & .53 \\
\hline \multicolumn{6}{|c|}{ Correlaciones entre Factores } \\
\hline I & 1 & & & & \\
\hline II & $0.76^{* * *}$ & 1 & & & \\
\hline III & $0.55^{* * *}$ & $0.61 * * *$ & 1 & & \\
\hline IV & $0.83^{* * *}$ & $0.86^{* * *}$ & $0.75^{* * *}$ & 1 & \\
\hline
\end{tabular}

$* * * p<.001$

con grados de libertad igual a las diferencias entre los grados de libertad asociado a cada modelo. Los resultados muestran que el modelo de 1 factor posee un ajuste estadísticamente peor que el de 2 factores (??2 $(1)=634.53, p=0.000)$. Lo mismo ocurre al comparar el modelo de 2 factores respecto del modelo propuesto $(? ? 2(5)=368,56, p=0.000)$.

Al analizar los parámetros del modelo propuesto (tabla 4), se aprecia que los indicadores de cada factor presentan cargas factoriales dentro del rango de .42 a .80 , todas estadísticamente significativas $(p<.001)$. Es relevante mencionar que sólo el $17 \%$ de la varianza del indicador laberintos es explicado por el factor II, siendo el indicador con menor porcentaje de varianza explicada en el modelo.

Las correlaciones entre los factores son moderada-alta, oscilando en el rango de 0.51 a .81 . El factor distractibilidad tiene una correlación alta $(r=.81, p<0.001)$ con el factor comprensión verbal -siendo la más elevada del modelo- y con organización espacial $(r=.74, p<0.001)$. Elevada es también la correlación entre los factores comprensión verbal y organización perceptual $(r=.72, p<0.001)$. Sólo el factor velocidad de procesamiento, presenta correlaciones moderadas con los factores comprensión verbal $(r=.51, p<$ $0.001)$ y con organización perceptual $(r=.65, p<0.001)$.

$\mathrm{Al}$ analizar cada rango etario se aprecia que la estructura factorial presenta un ajuste dado el tamaño muestral en todas ellos (índices de ajuste absoluto, ver Tabla 5). No obstante, en la muestra compuesta por niños de 6 a 7 años, los índices RMSEA, SNCF y los indicadores de ajuste incremental (índice de Tucker-Lewis, TLI e índice de ajuste normado, NFI) se encuentran cercanos a los límites de los criterios de referencia (RMSEA $<0.08$, NFI, TLI $>.90$, Hair et al., 2004). 
Tabla 7

Parámetros de la solución completamente estandarizada. Modelo factorial de la muestra 8 a 10 años

\begin{tabular}{|c|c|c|c|c|c|}
\hline Factor & I & II & III & IV & $R^{2}$ \\
\hline Información & $.75^{* * *}$ & & & & .57 \\
\hline Analogías & $.83 * * *$ & & & & .68 \\
\hline Vocabulario & $.85^{* * *}$ & & & & .72 \\
\hline Comprensión & $.69 * * *$ & & & & .48 \\
\hline \multicolumn{2}{|c|}{ Completación Figuras } & $.62 * * *$ & & &. .38 \\
\hline \multicolumn{2}{|c|}{ Ordenamiento Historias } & $.68^{* * *}$ & & & .46 \\
\hline \multicolumn{2}{|c|}{ Construcción Cubos } & $.78 * * *$ & & & 60 \\
\hline \multicolumn{2}{|c|}{ Ensamblaje Objetos } & $.72 * * *$ & & & .52 \\
\hline \multicolumn{2}{|l|}{ Laberintos } & $.45^{* * *}$ & & & .21 \\
\hline \multicolumn{2}{|l|}{ Claves } & $.67 * * *$ & & .45 & \\
\hline \multicolumn{2}{|c|}{ Búsqueda Símbolos } & & $.76^{* * *}$ & & .58 \\
\hline \multicolumn{2}{|c|}{ Retención Dígitos } & & & $.53 * * *$ & .28 \\
\hline \multicolumn{2}{|l|}{ Aritmética } & & & $.72 * * *$ & .52 \\
\hline \multicolumn{6}{|c|}{ Correlaciones entre Factores } \\
\hline I & 1 & & & & \\
\hline II & $.73^{* * *}$ & 1 & & & \\
\hline III & $.55^{* * *}$ & $.70 * * *$ & 1 & & \\
\hline IV & $.83^{* * *}$ & $.74 * * *$ & $.71 * * *$ & 1 & \\
\hline
\end{tabular}

$* * * p<.001$

Tabla 8

Parámetros de la solución completamente estandarizada. Análisis factorial confirmatorio para la muestra 11-13 años

\begin{tabular}{|c|c|c|c|c|c|}
\hline Factor & I & II & III & IV & $R^{2}$ \\
\hline Información & $.80^{* * *}$ & & & & .63 \\
\hline Analogías & $.79 * * *$ & & & & .63 \\
\hline Vocabulario & $.80 * * *$ & & & & .64 \\
\hline Comprensión & $.59 * * *$ & & & & .35 \\
\hline Completación & & $.54 * * *$ & & & .29 \\
\hline Ordenamiento & & $.61 * * *$ & & & .37 \\
\hline Construcción & & $.77 * * *$ & & & .60 \\
\hline Ensamblaje $\mathrm{Ot}$ & & $.70 * * *$ & & & .49 \\
\hline Laberintos & & $.35 * * *$ & & & .12 \\
\hline Claves & & $.65^{* * *}$ & & .43 & \\
\hline Búsqueda Sím & & & $.74 * * *$ & & .55 \\
\hline Retención Díg & & & & $.52 * * *$ & .27 \\
\hline Aritmética & & & & $.73 * * *$ & .53 \\
\hline \multicolumn{6}{|c|}{ Correlaciones entre Factores } \\
\hline I & 1 & & & & \\
\hline II & $.69^{* * *}$ & 1 & & & \\
\hline III & $.50 * * *$ & $.70 * * *$ & 1 & & \\
\hline IV & $.82 * * *$ & $.72 * * *$ & $.57 * * *$ & 1 & \\
\hline
\end{tabular}

$* * * p<.001$ 
Tabla 9

Parámetros de la solución completamente estandarizada. Análisis factorial confirmatorio para la muestra 14-16 años

\begin{tabular}{|c|c|c|c|c|c|}
\hline Factor & I & II & III & IV & $R^{2}$ \\
\hline Información & $.81 * * *$ & & & & .66 \\
\hline Analogías & $.80 * * *$ & & & & .64 \\
\hline Vocabulario & $.79 * * *$ & & & & 63 \\
\hline Comprensión & $.63 * * *$ & & & & .40 \\
\hline \multicolumn{2}{|c|}{ Completación Figuras } & $.57 * * *$ & & & .32 \\
\hline \multicolumn{2}{|c|}{ Ordenamiento Historias } & $.62 * * *$ & & & .38 \\
\hline \multicolumn{2}{|c|}{ Construcción Cubos } & $.77 * * *$ & & & .60 \\
\hline \multicolumn{2}{|c|}{ Ensamblaje Objetos } & $.72 * * *$ & & & .52 \\
\hline \multicolumn{2}{|l|}{ Laberintos } & $.35^{* * *}$ & & & .12 \\
\hline \multicolumn{2}{|l|}{ Claves } & $.60 * * *$ & & .36 & \\
\hline \multicolumn{2}{|c|}{ Búsqueda Símbolos } & & $.86^{* * *}$ & & .73 \\
\hline \multicolumn{2}{|c|}{ Retención Dígitos } & & & $.50 * * *$ & .25 \\
\hline \multicolumn{2}{|c|}{ Aritmética } & & & $.85^{* * *}$ & .72 \\
\hline
\end{tabular}

Correlaciones entre Factores

\begin{tabular}{ccccc}
\hline I & 1 & & & \\
II & $.70^{* * *}$ & 1 & & \\
III & $.45^{* * *}$ & $.57^{* * *}$ & 1 & 1 \\
IV & $.76^{* * *}$ & $.64^{* * *}$ & $.46^{* * *}$ & 1 \\
\hline
\end{tabular}

$* * * p<.001$

Los parámetros del modelo factorial propuesto para cada uno de los grupo etario (Tablas 6 a la tabla 9), muestran cargas factoriales en el rango de .35 a .86 (estadísticamente significativas a nivel $p<.001$ ). Coherente con los resultados de la muestra total el porcentaje de varianza del indicador laberintos explicados por el factor II es baja, alcanzando al $21 \%$ en la muestra de 8 a 10 años y un $12 \%$ en los mayores, de 11 a 16 años. Sólo en el grupo de menor edad, el factor II explica el $30 \%$ de la varianza del indicador.

En este rango etario se presentan dos diferencias adicionales respecto del patrón de la muestra total. A nivel de indicadores, solo el $24 \%$ de la varianza de "Claves" es explicada por el factor III. Segundo, en las correlaciones entre factores, la correlación más alta se establece entre factores organización perceptual y distractibilidad $(r=.86, p<0.001)$

Las diferencias en este tramo etario indicarían una indiferenciación de las habilidades, y probablemente sea indicador de resabios de pensamiento preoperatorio, que hace difícil la separación del razonamiento más abstracto, de la ejecución práctica para la resolución de problemas.

\section{Consistencia Interna de las Escalas}

La Tabla 10 presenta los coeficientes de consistencia interna para las subpruebas del WISC-III estandarizado en Chile y las escalas de C.I., para cada rango de edad, el promedio de los coeficientes para esos rangos, y el valor total. Se estimó la consistencia interna a través del coeficiente a de Cronbach. Para Claves y Búsqueda de Símbolos no pudo estimarse su consistencia interna por cuanto no presentan ítemes diferenciados sino que se establece un solo puntaje total cumplido un tiempo prefijado.
Los coeficientes de consistencia interna de las subpruebas son algo menores que los resultantes en el WISC-III original en Estados Unidos, salvo a la edad de 6 años (en Información, Comprensión y Completación de Figuras), a los 7 años (en Construcción con Cubos), a los 9 años (en Vocabulario), y a los 11 y 14 años (en Composición de Objetos).

$\mathrm{Al}$ igual que en la estandarización del WISC-III en Estados Unidos, en Chile la Escala Verbal posee una mayor consistencia interna que la Escala de Ejecución.

Como norma general, las consistencias internas observadas son buenas, aunque con algunas variaciones importantes a nivel de subescalas (desde 0.65 en Dígitos hasta 0.91 en Vocabulario) y escalas ( 0.75 ejecución hasta 0.85 Verbal).

\section{Conclusiones}

El presente estudio ha mostrado los principales resultados de la estandarización chilena del WISCIII. En estas conclusiones, volveremos sobre los principales resultados siguiendo el mismo esquema de los objetivos del estudio. Dado que el reporte de resultados de estudios de esta naturaleza suele ser hecho en un lenguaje muy técnico, no necesariamente comprensible del todo para los profesionales que aplican cotidianamente las pruebas, nos permitimos en estas conclusiones incorporar algunas explicaciones que permitan comprender las principales aportaciones y eventuales aplicaciones que tiene este nuevo instrumento de medición. 
Tabla 10

Consistencia interna de las escalas por edad y total

Edad en años

\begin{tabular}{|c|c|c|c|c|c|c|c|c|c|c|c|c|c|}
\hline $\begin{array}{l}\text { Subprueba } \\
\text { /escala }\end{array}$ & 6 & 7 & 8 & 9 & 10 & 11 & 12 & 13 & 14 & 15 & 16 & Promedio & Total \\
\hline Información & 0.75 & 0.73 & 0.76 & 0.75 & 0.73 & 0.79 & 0.72 & 0.79 & 0.78 & 0.77 & 0.76 & 0.76 & 0.90 \\
\hline Analogías & 0.77 & 0.74 & 0.78 & 0.77 & 0.74 & 0.77 & 0.69 & 0.70 & 0.72 & 0.75 & 0.73 & 0.74 & 0.87 \\
\hline Aritmética & 0.79 & 0.71 & 0.70 & 0.67 & 0.70 & 0.73 & 0.62 & 0.71 & 0.68 & 0.68 & 0.71 & 0.70 & 0.85 \\
\hline Vocabulario & 0.79 & 0.79 & 0.81 & 0.83 & 0.80 & 0.83 & 0.79 & 0.76 & 0.81 & 0.78 & 0.79 & 0.80 & 0.91 \\
\hline $\begin{array}{l}\text { Comprensión } \\
\text { Retención }\end{array}$ & 0.80 & 0.71 & 0.65 & 0.71 & 0.71 & 0.70 & 0.70 & 0.69 & 0.75 & 0.68 & 0.72 & 0.71 & 0.85 \\
\hline $\begin{array}{l}\text { Digitos } \\
\text { Completación }\end{array}$ & 0.45 & 0.50 & 0.51 & 0.47 & 0.55 & 0.61 & 0.58 & 0.62 & 0.60 & 0.65 & 0.64 & 0.56 & 0.65 \\
\hline $\begin{array}{l}\text { Figuras } \\
\text { Ordenamiento }\end{array}$ & 0.84 & 0.78 & 0.76 & 0.74 & 0.66 & 0.72 & 0.63 & 0.65 & 0.62 & 0.57 & 0.58 & 0.69 & 0.87 \\
\hline $\begin{array}{l}\text { Historias } \\
\text { Construcción }\end{array}$ & 0.70 & 0.65 & 0.70 & 0.59 & 0.70 & 0.69 & 0.58 & 0.66 & 0.70 & 0.62 & 0.64 & 0.66 & 0.79 \\
\hline $\begin{array}{l}\text { Cubos } \\
\text { Ensamblaje }\end{array}$ & 0.79 & 0.79 & 0.76 & 0.81 & 0.82 & 0.83 & 0.74 & 0.81 & 0.77 & 0.77 & 0.81 & 0.79 & 0.89 \\
\hline Objetos & 0.61 & 0.63 & 0.62 & 0.61 & 0.63 & 0.66 & 0.49 & 0.67 & 0.63 & 0.64 & 0.68 & 0.63 & 0.80 \\
\hline Laberintos & 0.75 & 0.70 & 0.64 & 0.64 & 0.52 & 0.63 & 0.49 & 0.50 & 0.57 & 0.59 & 0.51 & 0.59 & 0.75 \\
\hline $\begin{array}{l}\text { Escala Verbal } \\
\text { Escala }\end{array}$ & 0.85 & 0.80 & 0.86 & 0.85 & 0.87 & 0.87 & 0.81 & 0.82 & 0.85 & 0.85 & 0.86 & 0.85 & 0.85 \\
\hline Ejecución & 0.76 & 0.69 & 0.78 & 0.78 & 0.79 & 0.80 & 0.69 & 0.74 & 0.74 & 0.74 & 0.71 & 0.75 & 0.75 \\
\hline Escala Total & 0.88 & 0.84 & 0.88 & 0.88 & 0.88 & 0.88 & 0.84 & 0.84 & 0.87 & 0.86 & 0.86 & 0.86 & 0.87 \\
\hline
\end{tabular}

Respecto del proceso de adaptación y estandarización de la versión chilena del WISC-III

La presente investigación se basa en resultados de una muestra de 1924 niños chilenos de entre 6 y 16 años, de ambos sexos, seleccionados aleatoriamente en base a un muestreo estratificado por regiones. Tanto la magnitud de la muestra, como el hecho que se base en un muestreo estratificado y por regiones del país, nos permiten confiar en que las normas que se construyan en base a esta aplicación experimental, son confiables y representativas de las diferentes especificidades subculturales que puedan existir en el país. Es preciso destacar que la estandarización norteamericana fue realizada en una muestra de 2200 niños, esto es, un $10 \%$ más que la del presente estudio, para cubrir una población casi 20 veces mas numerosa. El proceso de estandarización siguió un proceso riguroso de adaptación de la versión argentina del test, estudio con jueces externos respecto de la pertinencia de los ítemes para la cultura chilena y adaptación de los reactivos que mostraron problemas en la aplicación piloto.
Es preciso destacar que tanto la aleatorización de la muestra, como el hecho que esta sea estratificada por regiones, dan una verdadera garantía que el instrumento ofrecido cumple con los estándares científicos necesarios para asegurar una evaluación justa y confiable por parte de aplicadores entrenados.

Respecto de las principales diferencias del WISCIII con el WISC-R

Un análisis comparativo de los contenidos del presente test con el preexistente, nos permiten garantizar que, a pesar de contar con un instrumento actualizado y renovado, éste conserva la misma estructura, principios y nomenclatura del instrumento reconocido como de uso estándar por los profesionales en Chile en los últimos 27 años (Simonetti \& Rosas, 1981; Ramírez, Cumsille \& Simonetti, 1996).

A nivel de los ítemes, las diferencias son variadas tanto en lo que respecta al contenido como a la cantidad de ellos en las distintas escalas. A nivel de las subescalas, la única diferencia realmente digna 
de destacar entre ambas pruebas, es la inclusión en el WISC III de una prueba totalmente nueva (Búsqueda de Símbolos). A nivel de las Escalas, estas se conservan igual, llamándose Escala Verbal y Escala de Ejecución. Tanto los procedimientos de cálculo de puntaje de las subescalas como de estimación de puntajes de C.I. se conservan sin variaciones, salvo algunas especificidades del cálculo de las bonificaciones por tiempo u otras en algunas de las escalas.

Un aspecto digno de destacar, es que el grado de dificultad de los ítemes que se conservaron sin modificación del WISC-R en la presente versión del WISC-III, disminuyó como norma general. Este resultado, consistente con la investigación que muestra un consistente aumento del C.I. de las poblaciones en el tiempo (Flynn, 1994, 1999), reafirma la necesidad de contar con un instrumento calibrado al nivel de las habilidades de las generaciones actuales. Es preciso destacar que de no hacerse esta calibración de manera oportuna, el test de WISC-R tenderá de manera inevitable a ser un instrumento poco discriminativo y confiable, ya que tenderá a sobreestimar las capacidades de los niños. En otras palabras, dado que las poblaciones son cada vez más capaces intelectualmente, el uso de instrumentos estandarizados obsoletos, hace que una cada vez más creciente mayoría, paradojalmente, se encuentre sobre la media de la población.

\section{Respecto de la Estructura Factorial y \\ Consistencia Interna de las Escalas}

La Estructura Factorial de un test de inteligencia hace referencia a la forma cómo sus diferentes subtest se agrupan en dimensiones comunes. En general, las pruebas de inteligencia están correlacionadas entre sí, unas más que otras. El análisis factorial es una técnica que permite descubrir la agrupación subyacente de las escalas en factores mas generales.

La estructura factorial del WISC-III permite sustentar una solución de cuatro factores correlacionados, coincidentes con los obtenidos en la muestra de estandarización norteamericana y argentina: Comprensión Verbal, Organización Perceptual, Velocidad de Procesamiento y Ausencia de Distractibilidad.

Respecto de la consistencia interna de las escalas, tradicionalmemente se aceptan valores superiores a 0.70 , medidos por alpha de Cronbach. Los resultados obtenidos en el estudio permiten concluir de manera bastante clara que las escalas de C.I. Verbal, de Ejecución y Total cumplen holgadamente este criterio. Lo mismo puede decirse las todas las subescalas, salvo Dígitos que está levemente por debajo de ese valor.

\section{Referencias}

Adriazola, A., Ascencio, S., Bernales, M., Carrasco, C., Keller, A., Polanco, F., Salas, C. \& Santelices, R. (1976). Estandarización de la Escala Revisada de Wechsler para la medición de la inteligencia en los niños (WISC-R). Memoria para optar al título de psicólogo. Pontificia Universidad Católica de Chile, Santiago, Chile.

American Psychological Association (1992). Ethical principles of psychologists and code of conduct. American Psychologist, 51, 644-648.

Calderón, M., Castillo, M., Mandujano, L., Pérez, C.\& Purcell, C. (1980). Estandarización de la Escala Revisada de Inteligencia de Wechsler para niños chilenos del Area Metropolitana (WISC-R). Tercera etapa. Memoria para optar al título de psicólogo. Pontificia Universidad Católica de Chile, Santiago, Chile.

Campazzo, E. (1962). Estandarización de la escala de Wechsler para la medición de la inteligencia en los niños (WISC). Memoria para optar al título de psicólogo. Pontificia Universidad Católica de Chile, Santiago, Chile.

Cañas, M., Colzani, M., Domínguez, A., Domínguez, M., Jorquera, K., Orpinas, P. \& Valdivieso, P. (1978). Adaptación, análisis estadístico y construcción de normas de la Escala Revisada de Inteligencia de Wechsler para niños entre 6 años 0 días y 9 años 11 meses 30 días (WISC$R$ ). Memoria para optar al título de psicólogo. Pontificia Universidad Católica de Chile, Santiago, Chile.

Cayssials, A. (1998). La Escala de Inteligencia WISC-III en la evaluación psicológica infanto-juvenil. Buenos Aires: Paidós.

Cohen, S. (1999). Niños maltratados: análisis de los aspectos cognitivos a través del WISC-III. Revista Iberoamericana de Diagnóstico y Evaluación Psicológica, 1, 53-63.

Flynn, J. (1994). IQ gains over time. En R. J. Sternberg (Ed.), Encyclopedia of human intelligence (pp. 617-623). New York: Macmillan.

Flynn, J. (1999). Searching for justice: The discovery of IQ gains over time. American Psychologist, 54, 5-20.

Goleman, D. (1996). La inteligencia emocional: ¿Por qué es más importante que el CI? Buenos Aires: Javier Vergara.

Hair, J., Anderson, R., Tatham, R., \& Black, W. (2004). Análisis multivariante $\left(5^{a}\right.$ Ed.). Madrid: Prentice Hall.

Horn, A. \& Michele, M. (1999). Stability of WISC-R and WISC-III IQs and subtest scores for a learning-disabled sample. Dissertation Abstracts International. Section B: The Sciences and Engineering, 60, 5-B, 2344.

Kaufman, A. (1975). Intelligence testing with Test of Psycholinguistic Abilities. Illinois: University of Illinois Press Urbana.

Kaufman, A. (1979/1982). Psicometría razonada con el $W I S C-R$ (traducido por F. López). México D. F.: El Manual Moderno.

Matarazzo, J. (1972). Wechsler's measurement and appraisal of adult intelligence ( $5^{a}$ Ed.). Baltimore: Williams \& Wilkins.

Ramírez, V. Cumsille, P. \& Simonetti, F. (1996). Proyecto Centro de Información y Evaluación Psicológica (manuscrito no publicado). Pontificia Universidad Católica de Chile, Santiago, Chile. 
Simonetti, F. \& Rosas, R. (1986) Diagnóstico de los objetivos y métodos de la evaluación del rendimiento intelectual en niños chilenos de 6 a 17 años. Revista Chilena de Psicología, 8, 25-32.

Fecha de recepción: Junio de 2004.

Fecha de aceptación: Marzo de 2007.
Wechsler, D. (1991/1997). Test de Inteligencia para Niños WISC-III. Manual (Traducción de Ofelia Castillo). Buenos Aires: Paidós. 